\title{
Asymptomatic Bacteriuria among Pregnant Women Referred to Outpatient Clinics in Sanandaj, Iran
}

\author{
Kalantar Enayat, Farhadifar Fariba, Nikkho Bahram
}

Department of Microbiology (KE), School of Medicine, Sanandaj University of Medical Sciences, Sanandaj, Iran, Department of Obstetrics (FF), Beassat Hospital, Sanandaj, Iran and School of Medicine (NB), Kurdistan University of Medical Sciences, Sanandaj, Iran

\begin{abstract}
Objectives: Determine the prevalence of asymptomatic urinary tract infection (AUTI) among pregnant women. We also determined the antibacterial susceptibility of the isolates to various antibiotics and associated risk factors in AUTI.

Materials and Methods: One thousand five hundred and five consecutive pregnant women were included in the study. Mid-stream urine specimen for complete examination of urine was obtained.

Results: Of 1505 pregnant women, $134(8.9 \%)$ had bacteriuria. The mean age of the all the pregnant women included in the study was 28.40 years with a standard deviation of 6.16. Age ranged from 15 to 45 years of age. The urine culture of the asymptomatic pregnant women (1505 cases) showed growth in only 134 cases $(8.9 \%)$. Escherichia coli was the commonest organism 79 (58.96\%) followed by CN Staphylococcus $22(16.8 \%)$ and S aureus $18(13.43 \%)$.

Escherichia coli, which comprised $58.96 \%$ (79) of the isolates, were $88.62 \%, 87.35 \%$, and $83.55 \%$ sensitive to cefotaxime, ciprofloxacin and cefotizoxime respectively. Similarly, E. coli were 89\%, 70\%, and 20\% resistant to ampicillin, cotrimoxazole, and nitrofurantoin respectively (OR 1.57 95\% CI 1.01, 2.44). After analyzing, four variables, hemoglobin levels seem to be independently associated with asymptomatic bacteriuria $(\mathrm{OR}=9.41(1.65-50.38)$.

Conclusion: Prevalence of asymptomatic bacteriuria among pregnant women was $8.9 \%$. The predominant organisms were Escherichia coli 58.96\% (79\%), followed by CN Staphylococcus 22 (16.8\%). Most strains of Escherichia coli showed that they were resistant to ampicillin, tetracycline and gentamicin.
\end{abstract}

Key words: urinary tract infection; pregnant women; symptoms

Int Braz. J Urol. 2008; 34: 699-707

\section{INTRODUCTION}

Urinary tract infection (UTI) is one of the most common reasons for people to seek medical consultation and is also one of the most frequently occurring nosocomial infections. UTI affects all age groups, but women particularly pregnant women are more susceptible than men, due to pregnancy, short urethra, easy contamination of urinary tract with fecal flora and various other reasons $(1,2)$.

Asymptomatic bacteriuria (ASB) is bacteriuria without apparent symptoms of urinary tract infections. The importance of ASB is a major risk factor for the development of UTI $(3,4)$.

In the past years, scientists have spent considerable time and effort investigating the frequency of occurrence and consequences of asymptomatic bacteriuria in pregnancy (5-8).

Few studies in Iran have shown that the prevalence of asymptomatic bacteriuria among pregnant women ranged from $6.1 \%$ to $10.9 \%$ (9-11).

Since isolated pathogens frequency and antimicrobial resistance rates can vary dramatically, even within the same countries, certain potentially resistant 
strains such as those causing asymptomatic urinary tract infections (AUTI) among pregnant women, require surveillance of the most common causative species $(12,13)$.

The prevalence rate of asymptomatic bacteriuria in pregnant women is comparable to the prevalence rate of non-pregnant women, indicating that pregnancy alone does not necessarily incline to the development of asymptomatic bacteriuria. It has been suggested that the frequency of bacteriuria increases by about $1 \%$ during pregnancy (6). The risk of acquiring bacteriuria increases with the duration of pregnancy from $0.8 \%$ of women with bacteriuria in the $12^{\text {th }}$ gestational week to $2 \%$ at the end of pregnancy (6).

There are a number of conditions associated with an increased prevalence of asymptomatic bacteriuria in pregnancy. Low socioeconomic status, sickle cell traits, diabetes mellitus and grand multiparity have been reported; each is associated with two-fold increase in the rate of bacteriuria (5).

A major study comparing normal and highrisk pregnant women reported $6.0 \%$ prevalence in healthy women; $12.2 \%$ rate in diabetic women and $18.7 \%$ in women with a previous history of urinary tract infection (5). Maternal anemia has been reported to be associated with both asymptomatic bacteriuria and pyelonephritis, but an association with covert bacteriuria has not yet been confirmed (14).

There is insufficient local data on asymptomatic bacteriuria among pregnant women in Sanandaj. The objectives of this study was to determine the prevalence of asymptomatic bacteriuria detected on the first pre-natal visit among pregnant women and to identify factors that increases the risk of developing asymptomatic bacteriuria in pregnant women.

\section{MATERIALS AND METHODS}

A total of 10 clinics from South, North, West, East and Central of Sanandaj city were randomly selected for this study to determine the prevalence of asymptomatic urinary tract infection (AUTI) among pregnant women. We also determined the antibacterial susceptibility of the isolates to various antibiotics and associated risk factors in AUTI.

All pregnant women consulting for their first pre-natal check- up, who were willing to participate, were included in the study. The following patients were excluded (a) patients with history of fever (b) patients with any two of the following genitourinary complaints: dysuria, urinary hesitancy, urgency, slow stream, incontinence, frequency, incomplete voiding and (c) patients with any intake of antibiotics for any indication during the current pregnancy.

One thousand five hundred and five consecutive pregnant women were included in the study. Mid-stream urine specimen for complete examination of urine was obtained. Asymptomatic Bacteriuria in pregnancy is defined clinically as: (a) $>100,000$ colony forming units of a single bacterial uropathogen per $\mathrm{mL}$ of midstream urine specimen and (b) the absence of symptoms attributable to urinary infection.

During the study period, all the urine samples were analyzed manually for culture and sensitivity using the semiquantitative calibrated loop method. Urine was inoculated on to blood agar and eosin methylene blue plates. The plates were incubated overnight. Next morning, a colony count was done, and interpreted according to our local interpretation guidelines. Bacteria were isolated and identified based on biochemical tests (15).

Antimicrobial susceptibility testing by disc diffusion was done according to the Clinical and Laboratory Standard Institute guidelines (16). This study was supported by grant from Kurdistan University of Medical Sciences.

Data was coded, computed and analyzed using SPSS version 11.5 and $p$ values $\leq 0.05$ were considered to be statistically significant.

\section{RESULTS}

Table-1 shows the demographic characteristics of pregnant women screened for asymptomatic bacteriuria. Of the 1505 pregnant women, 134 (8.9\%) had bacteriuria. The mean age of the all the pregnant women included in the study was 28.40 years with a standard deviation of 6.16. Age ranged from 15 to 45 years of age. 
Table 1 - Demographic characteristics of pregnant women screened for asymptomatic bacteriuria.

\begin{tabular}{lccc}
\hline Characteristics & Non-Bacteriuria & Bacteriuria & p Value \\
\hline Age (years) & & & \\
$\quad$ Mean (SD) & $28.4(6.16)$ & $27.7(6.0)$ & 0.267 \\
$\quad$ Range & $16-45$ & $15-41$ & \\
Pregnancy & & & 0.321 \\
$\quad$ Mean (SD) & $1.86(0.95)$ & $1.7(1.0)$ & \\
$\quad$ Range & $0.0-8.0$ & & \\
Parity & & $0.65(0.86)$ & 0.292 \\
$\quad$ Mean(SD) & $0.74(0.77)$ & $0.0-5.0$ & \\
$\quad$ Range & $0.0-4.0$ & $11.35(1.5)$ & 0.001 \\
Hemoglobin (g/dL) & $12.8(6.3)$ & $4.6-21.2$ & \\
$\quad$ Mean(SD) & $7.8-13.7$ & \\
$\quad$ Range & &
\end{tabular}

$S D=$ standard deviation

Escherichia coli was the most common organism $79(58.96 \%)$ followed by CN Staphylococcus 22 (16.8\%) and S aureus 18 (13.43\%) (Table-2).

Escherichia coli, which comprised 58.96\% (79) of the isolates, were $88.62 \%, 87.35 \%$, and 83.55 $\%$ sensitive to cefotaxime, ciprofloxacin and cefotizoxime respectively (Table-3).

After analysis, four variables (age, parity, gravity, and hemoglobin level): hemoglobin levels seem to be independently associated with asymptomatic bacteriuria $(\mathrm{OR}=9.41(1.65-50.38)$.

\section{COMMENTS}

Women with asymptomatic bacteriuria during pregnancy are more likely to deliver premature or lowbirth-weight infants and have a 20 - to 30-fold increased risk of developing pyelonephritis during pregnancy compared with women without bacteriuria (17).

This study revealed that prevalence of ASB among pregnant women was $8.9 \%$, which is similar to other reported studies, with minor differences $(8,10,18)$.

Several studies have demonstrated that the geographical variability of pathogens occurrence in case of UTI is limited by the predominance of Gram negative, usually Enterobacteriaceae and particularly
E. coli and Enterobacter spp., in various regions of the world and the resistance patterns of these organisms can vary significantly between hospital, countries and continents (19-22).

In this study the etiologic agent Escherichia coli $79(58.96 \%)$ was the most frequent which is in agreement with similar reported studies in our region as well as in other parts of the world $(3,8,10,20,21,23)$.

Gram-positive organisms have recently received more attention as causing bacteriuria and urinary tract infection. Although, they are seen in small numbers during pregnancy they are recognized as important causes of urinary tract infection. Our study findings of coagulase negative Staphylococcus

Table 2-Bacteriologic isolates from pregnant women with asymptomatic bacteriuria.

\begin{tabular}{lc}
\hline Organism (s) & N (\%) \\
\hline E. coli & $79(58.96)$ \\
Coagulase negative Staphylococcus & $22(16.8)$ \\
Staphylococcus aureus & $18(13.43)$ \\
Enterobacter spp & $10(7.46)$ \\
Klebsiella spp & $5(3.73)$ \\
Total & $134(100)$ \\
\hline
\end{tabular}


Asymptomatic Bacteriuria among Pregnant Women

Table 3 - In vitro antibiotics sensitivity pattern of isolated organisms.

\begin{tabular}{lccccccccccc}
\hline & Cip & Ct & Nor & Sxt & $\begin{array}{c}\text { Ctx } \\
\text { Antibiotics Sensitivity (\%) }\end{array}$ & $\begin{array}{c}\text { NA } \\
\text { Organism (s) }\end{array}$ & & \multicolumn{7}{c}{ Tet } & Fm & Am & Gm & Amk \\
\hline E. coli & 69 & 66 & 58 & 20 & 70 & 15 & 01 & 23 & 02 & 04 & 07 \\
& $(87.35)$ & $(83.55)$ & $(73.43)$ & $(25.32)$ & $(88.62)$ & $(18.99)$ & $(1.27)$ & $(29.12)$ & $(2.54)$ & $(5.07)$ & $(8.87)$ \\
CNS & 14 & 12 & 10 & 04 & 18 & 16 & 02 & 10 & 08 & & \\
& $(63.64)$ & $(54.55)$ & $(45.46)$ & $(18.20)$ & $(81.82)$ & $(72.73)$ & $(9.10)$ & $(45.46)$ & $(36.37)$ & ND & ND \\
S. aureus & 06 & & 15 & 04 & 15 & & 04 & 08 & 06 & & \\
& $(33.34)$ & $\mathrm{ND}$ & $(83.34)$ & $(22.23)$ & $(83.34)$ & $\mathrm{ND}$ & $(22.23)$ & $(44.45)$ & $(33.34)$ & $\mathrm{ND}$ & $\mathrm{ND}$ \\
Enterobacter sp & 08 & 07 & 04 & 07 & & 09 & 04 & 06 & 04 & 07 & 04 \\
& $(80.00)$ & $(70.00)$ & $(40.00)$ & $(70.00)$ & $\mathrm{ND}$ & $(90.00)$ & $(40.00)$ & $(60.00)$ & $(40.00)$ & $(70.00)$ & $(40.00)$ \\
Klebsiella sp & 04 & & & 01 & 03 & 01 & 03 & & 03 & 03 & 02 \\
& $(80.00)$ & $\mathrm{ND}$ & $\mathrm{ND}$ & $(20.00)$ & $(60.00)$ & $(20.00)$ & $(60.00)$ & $\mathrm{ND}$ & $(60.00)$ & $(60.00)$ & $(40.00)$ \\
\hline
\end{tabular}

Cip = ciprofloxacin; $C t=$ cefotizoxime; Nor = norfloxacin $;$ Sxt = cotrimoxazole $;$ Ctx $=$ cefotaxime, NA = nalidixic acid; Tet $=$ tetracycline; $\mathrm{Fm}=$ nitrofurantoin; $\mathrm{Am}=$ ampicillin, $\mathrm{Gm}=$ gentamicin, Amk = amikacin, $N D=$ not done, CNS $=$ coagulase negative Staphylococcus

(CNS) were the second most common urine isolate and are similar to the findings of Khattak et al. (23) and Abdullah et al. (24).

Regarding the antibiotics, E. coli exhibited $88.62 \%$ and $87.35 \%$ sensitivity to cefotaxime and ciprofloxacin respectively. In contrast, $73.43 \%$ and $79.74 \%$ were resistant to nalidixic acid and cotrimoxazole respectively and least susceptible to tetracycline, ampicillin, gentamicin and amikacin (Table-3). However, Shanson (25) reported the prevalence of resistance of urinary isolates to gentamicin was $2 \%$.

In view of changing pattern of bacterial resistance to common drugs, the importance of educating physicians to use these antibiotics for empiric therapy is important.

Factors proposed to affect the frequency of bacteriuria during pregnancy include multiparity, age, previous medical history of UTI, diabetes mellitus, anatomic urinary tract abnormalities, and socio-economic status $(3,13)$.

In this study, after analyzing, four variables such as age, hemoglobin, parity and gravity: hemoglobin levels hemoglobin levels seem to be independently associated with asymptomatic bacteriuria $(\mathrm{OR}=9.41(1.65-50.38)$ which is similar to other studies $(5,13)$. Although the proposed association between covert bacteriuria and anemia during pregnancy has not been confirmed, nevertheless, our study found hemoglobin is to be an independent risk factor. In West Africa, anemia in pregnancy results from multiple causes, including iron and folate deficiency; malaria and hookworm infestation; infections, such as HIV; and hemoglobinopathies (26).

Few recently studies also revealed that anemia continues to be a major health problem in many developing countries and is associated with increased rates of maternal and perinatal mortality, premature delivery, low birth weight, and other adverse outcomes (27).

\section{CONCLUSION}

Prevalence of asymptomatic bacteriuria among pregnant women was $8.9 \%$. The predominant organisms were Escherichia coli 58.96\% (79)\%, followed by CN Staphylococcus 22 (16.8\%). Most strains of Escherichia coli showed resistant to ampicillin, tetracycline and gentamicin. 


\section{ACKNOWLEDGEMENTS}

To Mrs. Kakaie S for reviewing the data coding and entry to computer. The Deanship of Scientific Research, Kurdistan University of Medical Sciences, provided financial support.

\section{CONFLICT OF INTEREST}

None declared.

\section{REFERENCES}

1. Gupta K, Sahm DF, Mayfield D, Stamm WE: Antimicrobial resistance among uropathogens that cause community-acquired urinary tract infections in women: a nationwide analysis. Clin Infect Dis. 2001; 33: 89-94.

2. Al-Dujiaily AA: Urinary tract infection during pregnancy in Tikrit. Med. J. Tikrit. 2000; 6: 220-4.

3. Al-Haddad AM: Urinary tract infection among pregnant women in Al-Mukalla district, Yemen. East Mediterr Health J. 2005; 11: 505-10.

4. Patterson TF, Andriole VT: Detection, significance, and therapy of bacteriuria in pregnancy. Update in the managed health care era. Infect Dis Clin North Am. 1997; 11: 593-608.

5. Kiningham RB: Asymptomatic bacteriuria in pregnancy. Am Fam Physician. 1993; 47: 1232-8.

6. Nicolle LE: Asymptomatic bacteriuria: when to screen and when to treat. Infect Dis Clin North Am. 2003; 17: 367-94.

7. Tugrul S, Oral O, Kumru P, Köse D, Alkan A, Yildirim G: Evaluation and importance of asymptomatic bacteriuria in pregnancy. Clin Exp Obstet Gynecol. 2005; 32: $237-40$.

8. Uncu Y, Uncu G, Esmer A, Bilgel N: Should asymptomatic bacteriuria be screened in pregnancy? Clin Exp Obstet Gynecol. 2002; 29: 281-5.

9. Mohammad M, Mahdy ZA, Omar J, Maan N, Jamil MA: Laboratory aspects of asymptomatic bacteriuria in pregnancy. Southeast Asian J Trop Med Public Health. 2002; 33: 575-80.

10. Hazhir S: Asymptomatic bacteriuria in pregnant women. Urol J. 2007; 4: 24-7.

11. Shirazi MH, Sadeghifard N, Ranjbar R, Daneshyar E, Ghasemi A: Incidence of Asymptomatic Bacteriuria
During Pregnancy. Pak. J. Biol. Sci. 2006; 9: 1514.

12. Boroumand MA, Sam L, Abbasi SH, Salarifar M, Kassaian E, Forghani S: Asymptomatic bacteriuria in type 2 Iranian diabetic women: a cross sectional study. BMC Womens Health. 2006; 6: 4

13. Bandyopadhyay S, Thakur JS, Ray P, Kumar R: High prevalence of bacteriuria in pregnancy and its screening methods in north India. J Indian Med Assoc. 2005; 103: 259-62.

14. Nicolle LE: Management of Asymptomatic UTIs in Women. Medscape Womens Health. 1996; 1: 4.

15. Forbes BA, Bailey \& Scott's Diagnostic Microbiology, 10th ed. St. Louis, Mosby. 1998; pp. 283-304.

16. Clinical and Laboratory Standard Institute. Performance Standards for Antimicrobial Disk Susceptibility Tests. NCCLS documents M 100 - SIS, 940 West Valley Road. Wayne, PA, 19087 USA, 2005.

17. Smaill F: Antibiotics for asymptomatic bacteriuria in pregnancy. Cochrane Database Syst Rev. 2001; (2): CD000490. Review. Update in: Cochrane Database Syst Rev. 2007; 2: CD000490.

18. Kiningham RB: Asymptomatic bacteriuria in pregnancy. Am Fam Physician. 1993; 47: 1232-8.

19. Fatima N, Ishrat S: Frequency and risk factors of asymptomatic bacteriuria during pregnancy. J Coll Physicians Surg Pak. 2006; 16: 273-5.

20. Kutlay S, Kutlay B, Karaahmetoglu O, Ak C, Erkaya S: Prevalence, detection and treatment of asymptomatic bacteriuria in a Turkish obstetric population. J Reprod Med. 2003; 48: 627-30.

21. Akinloye O, Ogbolu DO, Akinloye OM, Terry Alli OA: Asymptomatic bacteriuria of pregnancy in Ibadan, Nigeria: a re-assessment. Br J Biomed Sci. 2006; 63: $109-12$.

22. Teppa RJ, Roberts JM: The uriscreen test to detect significant asymptomatic bacteriuria during pregnancy. J Soc Gynecol Investig. 2005; 12: 50-3.

23. Khattak AM, Khattak S, Khan H, Ashiq B, Mohammad D, Rafiq M: Prevalence of asymptomatic bacteriuria in pregnant women. Pak. J. Med. Sci. 2006; 22: 162-6.

24. Abdullah AA, Al-Moslih MI: Prevalence of asymptomatic bacteriuria in pregnant women in Sharjah, United Arab Emirates. East Mediterr Health J. 2005; 11: 1045-52.

25. Shanson DC: Infection of the urinary tract. In: Microbiology in clinical practice, 2.nd. (ed.), Butterworth, London. 1989; pp. 430-50.

26. Mockenhaupt FP, Rong B, Günther M, Beck S, Till $\mathrm{H}$, Kohne E, et al.: Anaemia in pregnant Ghanaian 
women: importance of malaria, iron deficiency, and haemoglobinopathies. Trans R Soc Trop Med Hyg. 2000; 94: 477-83.
27. Dim CC, Onah HE: The prevalence of anemia among pregnant women at booking in Enugu, South Eastern Nigeria. MedGenMed. 2007; 9: 11-14

Accepted after revision: September 1, 2008

\section{Correspondence address:}

Dr. Kalantar Enayat

Department of Microbiology, School of Medicine

Kurdistan University of Medical Sciences

Sanandaj, Iran

Fax: + 00988716664649

E-mail: kalantar_enayat@yahoo.com

\section{EDITORIAL COMMENT}

Urinary tract infection (UTI) is one of the most frequently occurring nosocomial infections. UTI affects all age groups, but women particularly pregnant women are more susceptible than men, due to pregnancy, short urethra, easy contamination of urinary tract with fecal flora, immunodeficiency of the pregnancy and various other reasons. Several socio-demographic characteristics were found significantly associated with UTIs, such as age 30 years and more, illiterates and low educational level, low socio-economic level, unsatisfactory personal hygiene and use of underwear clothes other than cotton. Significant associations with UTIs were also found in multigravidae $4^{\text {th }}$ and more, those having more than one child and those who previously suffered UTIs. Authors of the present study demonstrated that hemoglobin levels might be independently associated with asymptomatic bacteriuria. Further investigation may associate this finding with the low socio-economic level. Urological conditions in pregnancy represent a major diagnostic and therapeutic challenge. As- ymptomatic bacteriuria and acute cystitis of pregnant women, even if uncomplicated and non-progressive are associated to poorer pregnancy prognosis and they need to be properly treated. Definition of the optimal antimicrobial agent for the treatment of asymptomatic bacteriuria or uncomplicated UTI in pregnant women is controversial. Among the most important factors in the choice of antimicrobial agent in a certain population of pregnant women to consider are the frequently isolated urinary pathogenic bacteria and microbial resistance. Until there are data from well-designed trials that establish the optimal duration of therapy for asymptomatic bacteriuria, standard treatment courses are recommended. 


\section{EDITORIAL COMMENT}

Screening for and treatment of asymptomatic bacteriuria is a standard procedure of obstetrical care and included in most antenatal guidelines. Untreated asymptomatic bacteriuria is a risk factor for pyelonephritis in pregnancy and associated with low birth weight infants (1). Screening for asymptomatic bacteriuria has been included as one of the most cost-effective strategies for maternal and neonatal health in developing countries in a detailed analysis of interventions to achieve the millennium development goals for health (2).

There are, however, difficulties in the widespread implementation of screening and treatment of asymptomatic bacteriuria. Educational programs should emphasize the importance of early antenatal care and healthcare providers need to be aware of the importance of asymptomatic bacteriuria. Facilities to culture the urine are often not available in under-resourced settings and alternative diagnostic tests that are less expensive, easier to implement and have been validated in these populations are urgently needed.

There is no clear consensus in the literature on either the duration of therapy or the choice of antibiotics and this study from the Kurdistan region of Iran confirms the importance of knowing local resistance patterns. The resistance to ampicillin and co-trimoxazole very high, but nitrofurantoin retained reasonable activity and most isolates were sensitive to ciprofloxacin. Where recent surveys of antibiotic susceptibility have been carried out, staggering rates of resistance to ampicillin and cotrimoxazole are often found, meaning that these less expensive antibiotics cannot be used for presumptive treatment unless antibiotic susceptibility testing of the organism can be routinely performed. For the women in this study, there are few oral alternatives available. While the organisms generally remained sensitive to one of the parenteral third generation cephalosporins, these agents are usually reserved to treat severe infections, and ciprofloxacin and the other quinolones are not usually recommended in pregnancy. Older agents, e.g. fosfomycin, may need to be reconsidered.

In this population, the prevalence of asymptomatic bacteriuria was within published ranges at $8.9 \%$. Interestingly, staphylococcal species made up $30 \%$ of the isolates with $13 \%$ of urinary isolates reported as Staphylococcus aureus, an organism that has not traditionally been thought of as a urinary pathogen. The importance of the reported increase in gram positive organisms associated with bacteriuria in pregnancy is uncertain. More research which includes pregnancy outcomes and a thorough microbiology work-up, with speciation of the organisms and additional susceptibility testing is needed.

Enayat et al., did not provide information regarding the management of their patients and whether effective treatment was provided. Despite almost uniform guidelines, there is little evidence of adherence to screening recommendations. Poor adherence with screening in indigenous communities in Australia has been proposed as one explanation for worse pregnancy outcomes in this population (3). Structural problems related to the provision of care in remote communities were identified as contributing factors. It is important that well-designed treatment studies for women in low and middle income countries are performed that can address the emerging problem of antimicrobial resistance and additional research on the implementation and outcome of programs to screen and treat pregnant women for asymptomatic bacteriuria in diverse settings is needed.

\section{REFERENCES}

1. Smaill F, Vazquez JC: Antibiotics for asymptomatic bacteriuria in pregnancy. Cochrane Database Syst Rev. 2007; CD000490.

2. Adam T, Lim SS, Mehta S, Bhutta ZA, Fogstad H, Mathai M, et al.: Cost effectiveness analysis of strategies for maternal and neonatal health in developing countries. BMJ. 2005; 331: 1107.

3. Bookallil M, Chalmers E, Andrew B: Challenges in preventing pyelonephritis in pregnant women in Indigenous communities. Rural Remote Health. 2005; 5: 39

Dr. Fiona Smaill

Department of Pathology and Molecular Medicine McMaster University Hamilton, Ontario, Canada Email:smaill@mcmaster.ca 


\section{EDITORIAL COMMENT}

In this edition of the International Braz $\mathrm{J}$ Urol authors Enayat, Fariba and Bahram report on their 2007 study of asymptomatic bacteriuria among pregnant women referred to outpatient's clinics in Sanandaj City, Iran. This is a well written, good study, on an important topic, handled in a scientifically rigorous manner.

The authors studied 1505 pregnant women from a total of ten clinics from South, North, West, East and Central portions of Sanandaj city, in the province of Kurdistan, Iran, in order to determine the prevalence of asymptomatic urinary tract infection. They found $8.9 \%$ had bacteriuria. Although the prevalence of asymptomatic bacteriuria in pregnant women is similar to the prevalence in non-pregnant women, the consequences of not treating are much more severe in pregnant women. According to the IDSA Guidelines for the Diagnosis and Treatment of Asymptomatic Bacteriuria in Adults (1), the appropriate screening test is a urine culture early on in pregnancy, preferably at week 16 of gestation. Since pregnant women with asymptomatic bacteriuria have increased risk of developing pyelonephritis, premature delivery, and birthing infants with low birth weight, it is recommended to treat asymptomatic bacteriuria with antimicrobials. The microbes cited by the IDSA Guidelines paper include E. coli, Enterobacteriaceae, coagulase-negative staphylococci, Enteroccocus, Group B streptococci, and Gardnerella vaginalis. Although many microbes can cause asymptomatic bacteriuria, the most common uropathogen reported by this Guidelines committee and found in the study by Enayat et al. was E. coli, although at a lower percentage in the Iranian study.

Similar standards of care have been implemented throughout the world. The study by Enayat et al. extends the observations of asymptomatic bacteria among pregnant women into Sanandaj, Iran. The study further clarifies that the same microbes around the world have different resistance patterns in the Kurdistan city where there studied occurred. The materials and methods of the study are consistent with the scientific methods used in the IDSA guidelines. In the IDSA guidelines, positive screen is considered to be $>100,000 \mathrm{cfu} / \mathrm{mL}$ in a sample of urine that has been collected appropriately to minimize contamination from a person without symptoms of urinary infection. The study maintained the same clinical standards as they consulted pregnant women without symptoms of urinary infection during their first pre-natal check-up. The study discovered that E. coli was the most common $(58.96 \%$ of pregnant women with asymptomatic bacteriuria), followed by coagulase negative Staphylococcus (16.8\%), and Staphylococcus aureus (13.43\%). However, the E. coli found was more resistant to the antibacterials that are recommended by the IDSA in their North American Guidelines. In Sanandaj, the E. coli were 89\%, 70\%, and $20 \%$ resistant to Ampicillin, Cotrimoxazole, and Nitrofurantoin respectively, while in North America, nitrofurantoin and sulfamethoxazole are prominently used to treat asymptomatic bacteriuria, because of the lower rates of resistance in the North American region. The study also indicated that E. coli in Sanandaj showed sensitivities to cefotaxime, ciprofloxacin, and cefotizoime at a rate of $88.62 \%, 87.355$, and $83.55 \%$ respectively. Hence, it seems that E. coli in Iran tend to be more resistant to common antimicrobials and require more aggressive treatment options. Known factors in causing increased antimicrobial resistance are a greater use of a given antibiotic in the region, and the prevalence of clonal group A, for examples. However, the question of why resistance is higher among E. coli in Sanandaj remains unclear, and was not the objective of this paper.

This study highlights the need for communities to be aware of their own local patterns of antimicrobial resistance. Proper antimicrobial treatment of women with asymptomatic bacteriuria must take into account the prevalence of different uropathogens, with the knowledge of the efficacy of different antimicrobials for such populations. This study is particularly interesting because the authors have helped identify the patterns of resistance in their community and have provided sensitivity data, which will be of value to physicians in this area. On a global scale, it is important that each community also be aware of their local patterns of resistance so as to facilitate the proper prescribing of antimicrobials, when indicated. 


\section{Asymptomatic Bacteriuria among Pregnant Women}

\section{REFERENCE}

1. Nicolle LE, Bradley S, Colgan R, Rice JC, Schaeffer A, Hooton TM; Infectious Diseases Society of America; American Society of Nephrology; American Geriatric
Society.Infectious Diseases Society of America guidelines for the diagnosis and treatment of asymptomatic bacteriuria in adults. Clin Infect Dis. 2005; 40: 643-54. Erratum in: Clin Infect Dis. 2005; 40: 1556.

\section{Dr. Richard Colgan \& Dr. Hengqi Zheng}

Department of Family and Community Medicine University of Maryland School of Medicine Baltimore, Maryland, USA E-mail:rcolgan@som.umaryland.edu 